\title{
HYBRID LINEAR/BILINEAR TIME-SCALE ANALYSIS
}

\author{
Martin Pasquier \\ Ecole Nationale Supérieure de Télécommunications \\ 46, rue Barrault, 75634 Paris Cedex 13, France \\ pasquier@email.enst.fr
}

\author{
Paulo Gonçalvès and Richard Baraniuk \\ Department of Electrical and Computer Engineering \\ Rice University, Houston, Texas 77005, USA \\ gpaulo@rice.edu, richb@rice.edu
}

\begin{abstract}
We introduce a new method for the time-scale analysis of nonstationary signals. Our work leverages the success of the "timefrequency distribution series / cross-term deleted representations" into the time-scale domain to match wide-band signals that are not well modeled in terms of time and frequency shifts. Using a wavelet decomposition and the Bertrand time-scale distribution, we locally balance linearity and bilinearity in order to provide good resolution while suppressing troublesome interference components. The theory of frames provides a unifying perspective and leads us to insights into the cross-term deleted representations.
\end{abstract}

\section{INTRODUCTION}

By displaying the time-varying frequency content of a nonstationary signal in terms of time and frequency variables, joint time-frequency and time-scale representations can reveal subtle features that remain hidden from other methods of analysis. Each type of representation matches a different class of signals. Timefrequency representations are covariant to time and frequency shifts and match signals with constant-bandwidth structure, such as narrow-band radar signals. Time-scale representations are covariant to time shifts and scale changes and match signals with proportional-bandwidth structure, such as wide-band sonar and acoustic signals. Many different representations exist, both linear and nonlinear.

Linear representations such as the short-time Fourier and Gabor time-frequency representations and the wavelet time-scale representation offer the benefit of simple interpretation at the expense of poor resolution. Bilinear representations such as the Wigner time-frequency distribution and the Bertrand time-scale distribution were developed as high resolution alternatives. While the nonlinearity of the bilinear distributions sharpens the local signal structure, it simultaneously generates interference between widely separated components that degrades the global structure. Traditionally, nonlinear interference due to these cross-components has been suppressed via smoothing over the time-frequency and time-scale planes $[1,2]$.

In [3, 4], Qian, Morris, and Chen introduced an alternative approach to time-frequency analysis that features an explicit and controllable linear vs. bilinear tradeoff. First the signal is represented in terms of a discrete sum of time-frequency concentrated atoms via a linear Gabor transform. Then the Wigner distribution, evaluated on

This work was supported by the National Science Foundation, grant no. MIP 9457438, and the Office of Naval Research, grant no. N00014-95-10849 . this linear signal decomposition rather than on the signal itself, separates into two distinct components: the Wigner auto-components of the atoms (the quasi-linear part of the representation) and the Wigner cross-components of the atoms (the bilinear part of the representation). By limiting the number of cross-components entering into the sum, we can locally control the degree of nonlinearity of the time-frequency representation and furthermore tune it for maximum concentration with minimum cross-components.

This time-frequency decomposition performs very well, but it is matched only to signals possessing a constant-bandwidth structure. In this paper, we extend the concept of hybrid linear/bilinear analysis to the time-scale plane. Our approach is based on the linear wavelet transform and the bilinear Bertrand distribution. In the process of our development, we gain new insights into the procedure of Qian, Morris, and Chen. In Section 2, we briefly review their approach to quasi-linearizing the Wigner distribution. In Section 3 , we transpose the problem of hybrid linear/bilinear analysis to time-scale and propose a frame-based solution. After discussing an implementation of this new method in Section 4, we close with conclusions.

\section{HYBRID TIME-FREQUENCY ANALYSIS}

A hybrid linear/bilinear system for time-frequency analysis consists of three components:

1. a bilinear time-frequency mapping,

2. a discrete linear signal decomposition based on time-frequency concentrated "atoms,"

3. a rule for determining which cross-components to include in the overall signal representation.

In [3, 4], Qian, Morris, and Chen utilize the bilinear Wigner distribution, a linear Gabor transform with a Gaussian window, and a Manhattan distance criterion.

\subsection{Wigner distribution}

The Wigner distribution is in many senses the central bilinear timefrequency distribution [1]. The cross-Wigner distribution of two signals $r$ and $s$ is defined as ${ }^{1}$

$$
W_{r, s}(t, f)=\int r^{*}\left(t-\frac{\tau}{2}\right) s\left(t+\frac{\tau}{2}\right) e^{-j 2 \pi f \tau} d \tau .
$$

When $s=r$, we have the Wigner distribution $W_{s}(t, f)$.

\footnotetext{
${ }^{1}$ Throughout this paper, integration bounds run from $-\infty$ to $+\infty$.
} 
The excellent time-frequency localization properties of the autocomponents of the Wigner distribution result from its bilinear, "matched filter" structure. Unfortunately, this bilinearity also results in cross-components, which impair its representation of multi-component signals. The Wigner distribution of the multicomponent signal $s(t)=\sum_{i=1}^{N} s_{i}(t)$ reads as

$$
W_{s}(t, f)=\sum_{i=1}^{N} W_{s_{i}}(t, f)+\sum_{i \neq j} 2 \operatorname{Re}\left\{W_{s_{i}, s_{j}}(t, f)\right\} .
$$

The first sum comprises the auto-components, the second the crosscomponents. Traditionally, Wigner distribution cross-components have been suppressed via smoothing over the time-frequency plane. Two-dimensional convolution yields a distribution in Cohen's class of time-frequency representations [1]; affine convolution yields a distribution in the affine class of time-scale representations [2].

In [3, 4], Qian, Morris, and Chen introduced an alternative approach to cross-component suppression that marries the decomposition (2) with the linear Gabor transform.

\subsection{Gabor transform}

The Gabor transform represents a signal $s$ in terms of timefrequency atoms $[5]$ :

$$
\begin{aligned}
s(t) & =\sum_{(m, n)} c_{m, n} \phi_{m, n}(t), \quad(m, n) \in \mathbb{Z}^{2}, \\
c_{m, n} & =\left\langle s, \widetilde{\phi}_{m, n}\right\rangle=\int s(t)\left(\widetilde{\phi}_{m, n}(t)\right)^{*} d t .
\end{aligned}
$$

The synthesis atoms

$$
\phi_{m, n}(t)=g\left(t-n t_{0}\right) e^{j 2 \pi m f_{0} t}, \quad t_{0} f_{0} \leq 1,
$$

are obtained by shifting one basic atom $g$ to various times $n t_{0}$ and frequencies $m f_{0}$ in the time-frequency plane. The analysis atoms $\widetilde{\phi}_{m, n}$, which are different from the synthesis atoms in general, comprise the dual basis, and the product $t_{0} f_{0}$ corresponds to the density of placement of the atoms in the time-frequency plane.

The Gaussian function is the natural choice for the basic atom $g$. Since it is the most concentrated signal in time-frequency, the associated Gabor representation is as local as possible. Furthermore, the Wigner distribution of a Gaussian function is strictly positive and so has a correct energetic interpretation. The key drawback to Gaussian synthesis atoms is that for time-frequency sampling densities $t_{0} f_{0}$ close to unity, the corresponding dual basis functions have extremely poor localization properties (Balian-Low theorem $[5, \mathrm{p} .108]$ ) plus a spiky structure that does not allow a numerically stable reconstruction. Qian and Chen deal with this problem by in* troducing redundancy $\left(t_{0} f_{0} \ll 1\right)$ in the Gabor transform until both the basis and dual basis atoms resemble shifted, modulated Gaussians [4]. They term such a representation an "orthogonal-like" Gabor decomposition.

\subsection{Hybrid System}

Using Gaussian time-frequency atoms as building blocks, Qian, Morris, and Chen substitute (3) into (1) to decompose the Wigner distribution into a time-frequency distribution series - a sum of auto- and cross-Wigner distributions $[3,4]$

$$
\begin{aligned}
& W_{s}(t, f)=\sum_{(m, n)}\left|c_{m, n}\right|^{2} W_{\phi_{m, n}}(t, f) \\
& +\sum_{(m, n) \neq\left(m^{\prime}, n^{\prime}\right)} c_{m, n} c_{m^{\prime}, n^{\prime}}^{*} W_{\phi_{m, n}, \phi_{m^{\prime}, n^{\prime}}}(t, f)
\end{aligned}
$$

The first sum corresponds to the "quasi-linear part" of the representation; it is manifestly positive and indicates the gross features of the signal. The second sum corresponds to the "bilinear part" of the representation; it takes on both positive and negative values. Locally it serves to concentrate the features of the signal in the representation, while globally it creates interference components between nonadjacent portions of the signal.

By limiting the range of the second sum to include only $(m, n)$ close to $\left(m^{\prime}, n^{\prime}\right)$, we can retain the useful, local nonlinear interactions but suppress the noxious, global interactions. Given the circular or elliptical symmetry of the Gaussian atoms of the Gabor decomposition, a Euclidean distance metric seems natural to measure closeness. For computational simplicity, Qian and Chen approximate this measure with an $l^{\infty}$ metric (the so-called "Manhattan distance") $D_{\mathrm{M}}\left[(m, n),\left(m^{\prime}, n^{\prime}\right)\right]=\left|m-m^{\prime}\right|+\left|n-n^{\prime}\right|$. The hybrid linear/bilinear time-frequency representation of Qian, Morris, and Chen then corresponds to (5), but with the second sum restricted to component pairs such that $D_{\mathrm{M}}\left[(m, n),\left(m^{\prime}, n^{\prime}\right)\right] \leq \delta$. The threshold $\delta$ controls the localization vs. interference tradeoff.

\section{HYBRID TIME-SCALE ANALYSIS}

The hybrid linear/bilinear time-frequency representation of Qian, Morris, and Chen performs very well, but it matches only signals with a constant-bandwidth structure. For signals exhibiting a proportional bandwidth, wide-band structure, a time-scale analysis is more appropriate. To transfer the linear/bilinear decomposition concept from the time-frequency plane to the time-scale plane, we must find wide-band substitutes for the Wigner distribution, Gabor transform, Gaussian atom, and Euclidean distance.

\subsection{Bertrand Distribution}

The unitary Bertrand distribution can be considered as a central bilinear time-scale distribution [6]. The cross-Bertrand distribution of two signals $r$ and $s$ is defined in terms of their Fourier transforms $R$ and $S$ as

$$
B_{r, s}(t, f)=f \int R(\lambda(u) f) S^{*}(\lambda(-u) f) e^{j 2 \pi t f u} \mu(u) d u,
$$

with $\lambda(u)=\frac{u}{1-e^{-u}}$ and $\mu(u)=\sqrt{\lambda(u) \lambda(-u)}$. When $S=R$, we have the Bertrand distribution $B_{s}(t, f)$. Like the Wigner distribution, the Bertrand distribution inherits both its good localization and problematic cross-components [7] from its bilinear structure.

\subsection{Wavelet Transform}

The wavelet transform plays a role analogous to the Gabor transform in time-scale analysis. The wavelet transform also represents signals in terms of time-frequency atoms [5]:

$$
s(t)=\sum_{(m, n)} d_{m, n} \psi_{m, n}(t), \quad(m, n) \in \mathbb{Z}^{2},
$$




$$
d_{m, n}=\left\langle s, \widetilde{\psi}_{m, n}\right\rangle .
$$

Given a bandpass mother wavelet function $h$ centered at frequency $f_{c}$, we create the synthesis atoms

$$
\psi_{m, n}(t)=a_{0}^{-m / 2} h\left(a_{0}^{-m} t-n t_{0}\right),
$$

by shifting and scaling $h$ to various times $n t_{0} a_{0}^{m}$ and frequencies $a_{0}^{-m} f_{c}$ in the time-frequency plane. The proportional-bandwidth analysis of the wavelet transform matches wide-band signals. 41]

For the mother wavelet $h$, we choose the Klauder function [5, p.

$$
H(f)=f^{\beta} e^{-\alpha f}, \quad \alpha>0, \beta>0, f>0,
$$

expressed here in the frequency domain. The parameter $\beta$ controls the $Q$ factor of the wavelet, while $\alpha$ controls its extent. The properties of this function in time-scale mirror those of the Gaussian in time-frequency. First, the Klauder function is the most concentrated signal in time-scale, meaning that the associated wavelet representation is as local as possible. Second, the Bertrand distribution of a Klauder wavelet is positive, giving it a correct energetic interpretation [8]. The key drawback to Klauder synthesis atoms is that for low time-scale sampling densities the corresponding dual basis functions have poor localization properties. To better understand the links between bases and dual bases using the Klauder wavelet, we turn now to the theory of frames.

\subsection{Frames}

A frame is a family of functions $\left\{\gamma_{m, n}\right\}$ in a Hilbert space with the property that there exist two frame bounds $0<A \leq B<\infty$ such that for all signals $s$ in the space [5]

$$
A\|s\|^{2} \leq \sum_{(m, n)}\left|\left\langle s, \gamma_{m, n}\right\rangle\right|^{2} \leq B\|s\|^{2}
$$

The frame bounds can be estimated numerically given the frame. Depending on their values, we can distinguish several different categories of frames:

1. When $A=B$, we have a tight frame and the signal representation

$$
s(t)=A^{-1} \sum_{(m, n)}\left\langle s, \gamma_{m, n}\right\rangle \gamma_{m, n}(t) .
$$

The value $A$ measures the degree of redundancy of a tight frame. In particular, when $A=B=1$, the family $\left\{\gamma_{m, n}\right\}$ corresponds to an orthonormal basis.

2. When $A \approx B$, we have a snug frame. In this case, the signal representation (10) holds to a close approximation.

3. When $A \neq B$, the signal representation (10) does not hold. In this case, we must construct a different dual frame for analysis.

The theory of frames furnishes an elegant characterization of the Gabor and wavelet transforms. In particular, the concept of a snug frame provides insight into the "orthogonal-like" Gabor decomposition introduced in Section 2.2 [4]. Numerical computation of the frame bounds for $g$ Gaussian and $t_{0} f_{0}=0.25$ as suggested in [4] yields $B / A \approx 1.06$ and $A \approx 4$, meaning that at "four-times oversampling," we can set $\widetilde{\phi}_{m, n}=\phi_{m, n}$ in (4) to a close approximation.

In the wavelet case, using a Klauder function with $\beta=100$ and $\alpha=250$ on the normalized frequency axis $0<f<1$, we
Table 1: Frame bounds for wavelet frames generated using a Klauder wavelet $(\beta=100$ and $\alpha=250)$. We fix the time-step parameter at $t_{0}=1$ and vary the scale parameter $a_{0}$.

\begin{tabular}{|c||c|c|c|}
\hline$a_{0}$ & $A$ & $B$ & $B / A$ \\
\hline \hline 1.44 & 0.43 & 6.28 & 14.3 \\
\hline 1.27 & 2.82 & 6.31 & 2.24 \\
\hline 1.15 & 7.86 & 8.07 & 1.02 .7 \\
\hline 1.14 & 8.44 & 8.56 & 1.013 \\
\hline 1.13 & 9.09 & 9.14 & 1.006 \\
\hline
\end{tabular}

obtain a snug frame at approximately eight times the redundancy of a wavelet orthonormal basis (see Table 1).

For computational purposes, it is convenient to choose the scale parameter $a_{0}=2$. However, to obtain a snug frame with the Klauder wavelet, we must in turn set $t_{0}<1$, which would lead to problematic non-integer time shifts $n t_{0}$ in our atoms. One way around this difficulty is the concept of voices [5]. The basic idea is to interlace several (not necessarily snug) frame decompositions to create one equivalent snug decomposition. In our case, the mother wavelet for each of our $J$ voices is obtained from the Klauder function (9) by the scaling

$$
h^{\nu}(t)=2^{-\nu / J} h\left(2^{-\nu / J} t\right), \quad \nu=0, \ldots, J-1 .
$$

In Table 2, we give the frame bounds for several different $J$-voice wavelet frames constructed using $t_{0}=1, a_{0}=2$, and a Klauder function with parameters $\beta=100$ and $\alpha=250$. We chose $J=5$ for our final implementation.

Table 2: Frame bounds for wavelet frames generated using a Klauder wavelet $(\beta=100$ and $\alpha=250)$. We fix $t_{0}=1, a_{0}=2$, and vary the number of voices $J$.

\begin{tabular}{|c||c|c|c|}
\hline$J$ & $A$ & $B$ & $B / A$ \\
\hline \hline 2 & 1.4 & 14.2 & 10.0 \\
\hline 3 & 7.5 & 14.3 & 1.90 \\
\hline 4 & 13.4 & 15.6 & 1.16 \\
\hline 5 & 17.9 & 18.4 & 1.02 \\
\hline 10 & 36.3 & 36.3 & 1.00 \\
\hline
\end{tabular}

From Table 1, we know that we can obtain a snug frame using $t_{0}=1, a_{0}=1.13$, and $J=1$. Since $1.13^{5} \approx 2$, it is no surprise that we can also achieve a snug frame using $t_{0}=1, a_{0}=2$, and $J=5$.

\subsection{Hybrid System}

Using Klauder wavelet atoms as building blocks, we can decompose the Bertrand distribution into a sum of auto- and crossBertrand distributions. By limiting the interaction of the atoms according to their distance, we can both retain the (useful) local nonlinear interactions and suppress the (nuisance) global interactions. Denoting the distance metric by $D_{\mathrm{L}}$, we have

$$
\begin{aligned}
& \widehat{B}_{s}(t, f)=\sum_{(m, n)}\left|d_{m, n}\right|^{2} B_{\psi_{m, n}}(t, f) \\
+ & \sum_{D_{\mathrm{L}}\left[(m, n),\left(m^{\prime}, n^{\prime}\right)\right]<\delta} d_{m, n} d_{m^{\prime}, n^{\prime}}^{*} B_{\psi_{m, n}, \psi_{m^{\prime}, n^{\prime}}}(t, f) .
\end{aligned}
$$

The natural distance measure for time-scale analysis is the Lobachevsky metric [9]. With this measure, the distance between 
two wavelet atoms at $(m, n)$ and $\left(m^{\prime}, n^{\prime}\right)$ is defined as

$$
\begin{aligned}
& D_{\mathrm{L}}\left[(m, n),\left(m^{\prime}, n^{\prime}\right)\right]= \\
& \quad \cosh ^{-1}\left\{1+\frac{n n^{\prime}}{2 f_{c}^{2} a_{0}^{m+m^{\prime}}}\left[t_{0}^{2}\left(n a_{0}^{m}-n^{\prime} a_{0}^{m^{\prime}}\right)^{2}+\left(a_{0}^{m}-a_{0}^{m^{\prime}}\right)^{2}\right]\right\},
\end{aligned}
$$

with $f_{c}$ a reference frequency. In contrast to the time and frequency shift invariant Euclidean and Manhattan distances, the Lobachevsky distance is time-shift and scale invariant.

\section{IMPLEMENTATION}

The time-scale distribution series (11) has been implemented and performs well. However, lack of a closed form expression for the Bertrand distribution of the Klauder function limits the computational efficiency of the technique. For applications in which computation and storage are at a premium, we have developed an approximation to (11) with the following justification:

1. Even when the signal we analyze is wide-band, each local crossBertrand distribution involved in (11) is reasonably narrow-band given the narrow bandwidth of the Klauder wavelets and the fact that they interact at small distances only. Thus, since the Bertrand and Wigner distributions resemble one another closely for narrow-band signals [7], it is reasonable to replace the Bertrand distribution in (11) with the Wigner distribution.

2. For large values of $\beta$, the Klauder wavelet can be approximated using a modulated Gaussian (Morlet) wavelet $r(t)=$ $e^{-t^{2} / 2 \sigma^{2}} e^{i 2 \pi f_{c} t}$ with $\sigma=\alpha /(\pi * \sqrt{2 \beta+1})$ and $f_{c}=(2 \beta+$ 1) $/(2 \alpha)$.

With the change to a Wigner distribution and Morlet wavelet analysis, we obtain closed form expressions for the auto- and crossWigner distributions in the approximation to (11). The Wigner distribution of a Morlet wavelet is the 2-d Gaussian $W_{r}(t, f)=$ $2 \sigma \sqrt{\pi} \exp \left(-t^{2} / \sigma^{2}\right) \exp \left(-\left(2 \pi \sigma\left(f-f_{c}\right)\right)^{2}\right)$. The cross-Wigner distribution between a Morlet wavelet $r(t)$ and a shifted and scaled version of itself $q(t)=a_{0}^{-1 / 2} r\left(\left(t-t_{0}\right) / a_{0}\right)$ is given by

$$
\begin{aligned}
& W_{r, q}(t, f)=\sqrt{\frac{\pi}{C_{1}}} \exp \left\{\frac{\left(C_{2}+j C_{3}\right)^{2}}{4 C_{1}}\right\} \\
& \quad \times \exp \left\{-\frac{t^{2}}{2 \sigma^{2}}-\frac{\left(t-t_{0}\right)^{2}}{2 \sigma^{2} a_{0}^{2}}\right\} \exp \left\{j 2 \pi f_{0}\left(t-\frac{t-t_{0}}{a_{0}}\right)\right\},
\end{aligned}
$$

with $C_{1}=\frac{1}{8 \sigma^{2}}\left(1+1 / a_{0}^{2}\right), C_{2}=-\frac{1}{2 \sigma^{2}}\left(t-\left(t-t_{0}\right) / a_{0}^{2}\right)$ and $C_{3}=\pi\left(f_{0}+f_{0} / a_{0}-2 f\right)$. The results obtained using (11) and its Wigner/Morlet approximation show only very slight differences.

Figure 1 illustrates the performance of the hybrid linear/bilinear time-scale distribution on a synthetic test signal. This new method for time-scale analysis combines the high resolution of the Bertrand and Wigner distributions with the quasi-linearity of the scalogram (the squared magnitude of the wavelet transform):

\section{CONCLUSIONS}

In this paper, we have developed a new approach to time-scale analysis that offers an easily controlled tradeoff between linearity and bilinearity. Throughout our development, we have stressed the use of the right ingredients for time-scale analysis. The Bertrand distribution, wavelet frame with minimum uncertainty Klauder wavelet, and Lobachevsky time-scale distance combine to form a representation capable of matching a broad class of wide-band signals.

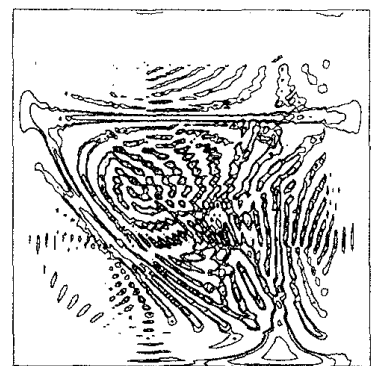

(a)

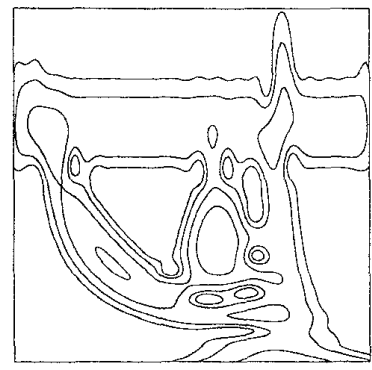

(c)

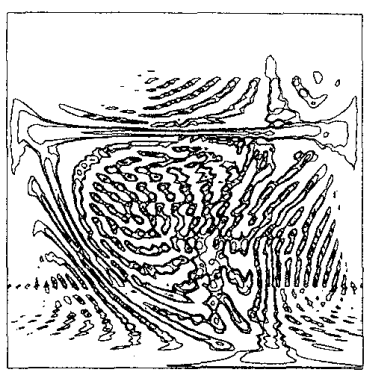

(b)

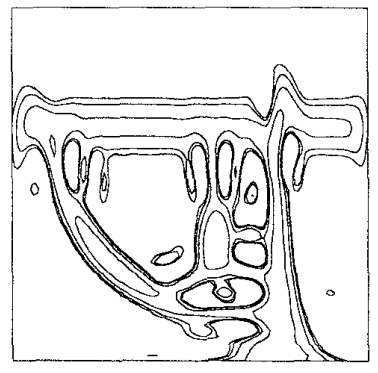

(d)
Figure 1: Time-frequency and time-scale distributions of a test signal composed of a sinusoid, a hyperbolic chirp, a Klauder wavelet pulse, and a local singularity of the form $\left|t-t_{0}\right|^{-0.15}$. Horizontal axis corresponds to time, vertical axis to frequency. (a) Wigner distribution. (b) Bertrand distribution (c) Scalogram (squared magnitude of the continuous wavelet transform). (d) New hybrid linear/nonlinear time-scale representation with interaction distance $\delta=0.5$.

\section{REFERENCES}

[1] L. Cohen, Time-Frequency Analysis. Englewood Cliffs, NJ: Prentice-Hall, 1995.

[2] O. Rioul and P. Flandrin, "Time-scale energy distributions: A general class extending wavelet transforms," IEEE Trans. Signal Processing, vol. 40, pp. 1746-1757, July 1992.

[3] S. Qian and J. M. Morris, "Wigner distribution decomposition and cross-terms deleted representation," Signal Processing, vol. 27, pp. 125-144, May 1992.

[4] S. Qian and D. Chen, "Decomposition of the Wigner distribution and time-frequency distribution series," IEEE Trans. Signal Processing, vol. 42, pp. 2836-2842, Oct. 1994.

[5] I. Daubechies, Ten Lectures on Wavelets. New York: SIAM, 1992.

[6] J. Bertrand and P. Bertrand, "A class of affine Wigner functions with extended covariance properties," J. Math. Phys., vol. 33, pp. 2515-2527, July 1992.

[7] P. Flandrin and P. Gonçalvès, "Geometry of affine timefrequency distributions," J. Appl. Comp. Harmonic Anal., vol. 3, pp. 10-39, Jan. 1996.

18] P. Flandrin, personnal communication.

[9] I. Daubechies, J. R. Klauder, and T. Paul, "Wiener measures for path integrals with affine kinematic variables," J. Math. Phys., vol. 28, pp. 85-102, Jan. 1987. 